\title{
Acetabular Reconstruction Using Massive Allografts for Combined Cavitary and Segmental Defects
}

\author{
Montalti $\mathrm{M}^{*}$, Guerra G and Giardina F \\ Hip and Knee Prosthetic Department, Laboratorio di Tecnologia Medica, Rizzoli Orthopaedic Institute, Bologna, Italy
}

${ }^{*}$ Corresponding author: Montalti M, M.D., Hip and Knee Prosthetic Department, Laboratorio di Tecnologia Medica, Rizzoli Orthopaedic Institute, Bologna, Italy Tel: +390516366962, Fax: +3990516366416, E-mail: maurizio.montalti@ior.it

Citation: Montalti M, Guerra G, Giardina F (2015) Acetabular Reconstruction Using Massive Allografts for Combined Cavitary and Segmental Defects. SAJ Cas Rep 2: 201. doi: 10.18875/2375-7043.1.401

Article history: Received: 05 January 2015, Accepted: 11 May 2015, Published: 13 May 2015

\section{Abstract}

Acetabular reconstruction in cavitary, segmental defects and pelvic discontinuity is a technically challenging surgical procedure. Today in orthopaedic surgery there are some available solutions to face this challenge: acetabular reinforcement devices with morselized or bulk grafts, Jumbo hemispherical cups, oblong or bilobed-shaped cups and anti-protrusio cages with morselized bone graft. In this manuscript we described two cases of pelvic discontinuity treated with bulk bone grafts and hemispherical press-fit cups in our Institution.

Keywords: Acetabular reconstruction, Radiographs, CT scan

\section{Introduction}

Aseptic loosening of an acetabular cup is often associated to extensive bone loss. Acetabular reconstruction in combined cavitary and segmental defects or in pelvic discontinuity is a technically challenging operation. It is necessary to build a stable support that could offer a long-term stability to the acetabular component.

To face this problem there are some available solutions: acetabular reinforcement devices with morselized or bulk grafts, Jumbo hemispherical cups, oblong or bilobed-shaped cups and anti-protrusio cages with morselized bone graft.

We reviewed two cases of important combined cavitary and segmental defects treated with bulk bone grafts and hemispherical press-fit cups in our Institution. The patients and their families were informed that data from the case would be submitted for publication, and gave their consent.

\section{Case 1}

A 82-year-old woman presented to our department in June 2011 with increasing pain in the right hip joint, limp and inability to walk. She had total hip arthroplasty in 1983 (diagnosis leading to primary arthroplasty was osteoarthritis secondary to developmental hip dysplasia). In 2002 she was treated of revision surgery due to aseptic loosening of the acetabular component. A stemmed acetabular cup (McMinn ${ }^{\circ}$ cup, Link UK) with morselized bone graft was implanted.

At physical examination the right hip had 70 degrees of flexion, minimal internal and external rotation and 15 degrees of abduction. There was a leg length discrepancy; the right affected side had become shortened by $2.5 \mathrm{~cm}$. Blood inflammatory markers were all within normal limits. Anterior-posterior and lateral-lateral views of the hip showed loosening of the acetabular and femoral component with superior migration $>2 \mathrm{~cm}$ and severe bone loss. CT scan of the pelvis revealed two large defects involving medial and superior wall.

Total bone scintigraphy and scintigraphy with labelled leukocytes did not show increased uptake of the isotopes in the right hip joint. From the Paprosky classification system[1] the acetabular defect was classified type III B with severe bone loss and impairment of the integrity of supporting structure. Kohler's line was not intact.

The direct lateral approach with the patient in a supine position was used. The failed implant and the cement (from inside femoral canal) were removed. Intraoperative samples for bacterial culture were negative. 
The acetabular defect was composed by two large cavities involving superior and medial wall. After appropriate preparation of the socket site (lodging), morselized allograft (40 gr.) and two femoral heads were impacted. A 62-mm uncemented porouscoated anatomic cup (Trilogy ${ }^{\oplus}$ Acetabular Hip System, Zimmer, Warsaw, Indiana USA) was implanted with a press-fit . Two supplementary acetabular screws were used to increase implant stability. A stable reduction was achieved with a 32-mm diameter metal femoral head. (Figure $1 \mathrm{a}, \mathrm{b}, \mathrm{c}$ ) (Figure 2) Full weight-bearing was precluded for 6 months after surgical procedure. At oneyear postoperative radiographs the cup was stable, the graft remodeled and the anatomy restored.
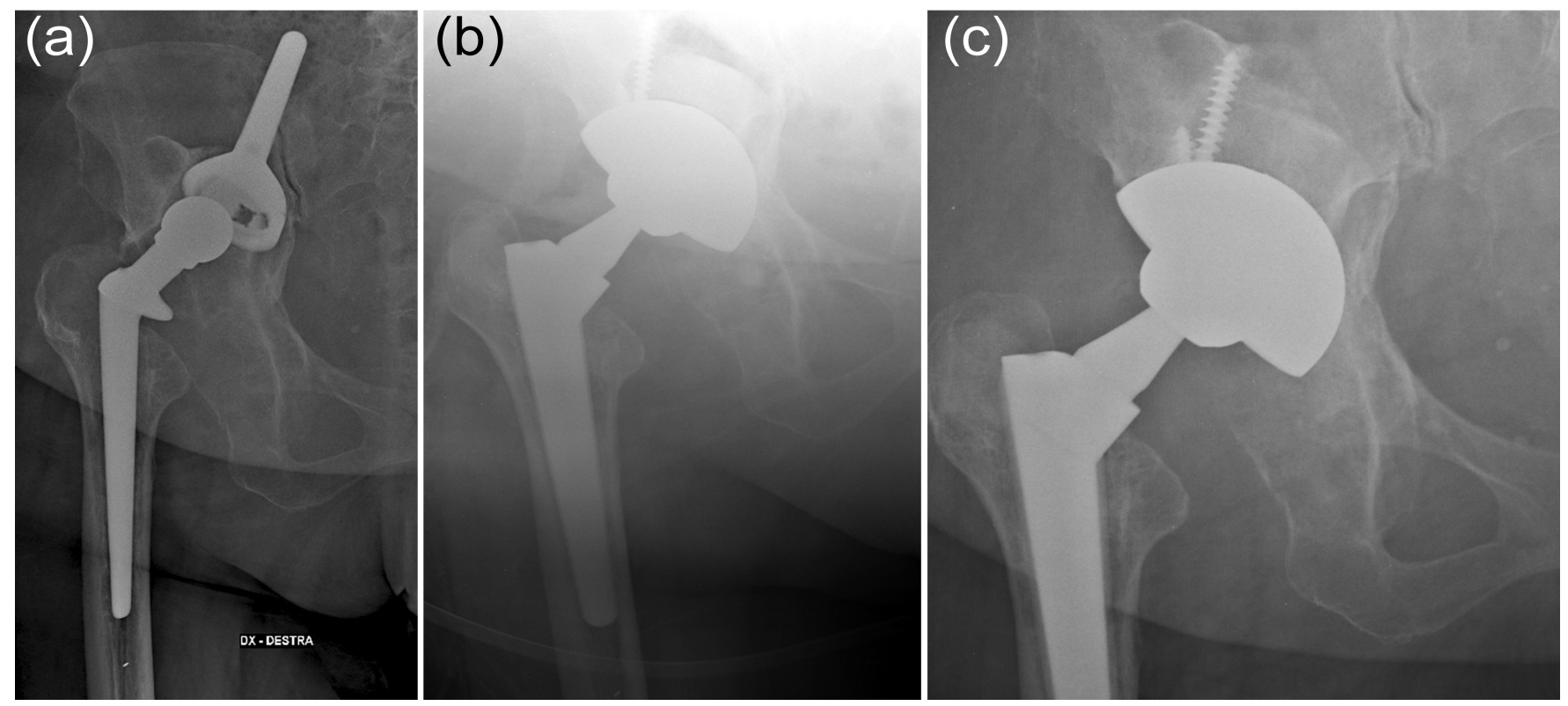

Figuure1: a. Anterior-posterior view of the pelvis showed loosening of the stemmed acetabular cup with superior migration. The medial and superior walls are involved by large bone defects. b. A-P radiograph showed reconstruction of the defect with two impacted femoral heads, morselized allografts and uncemented porous-coated anatomic cup. c. At 1 year follow-up A-P radiographs of the pelvis showed no evidence of graft resorption.

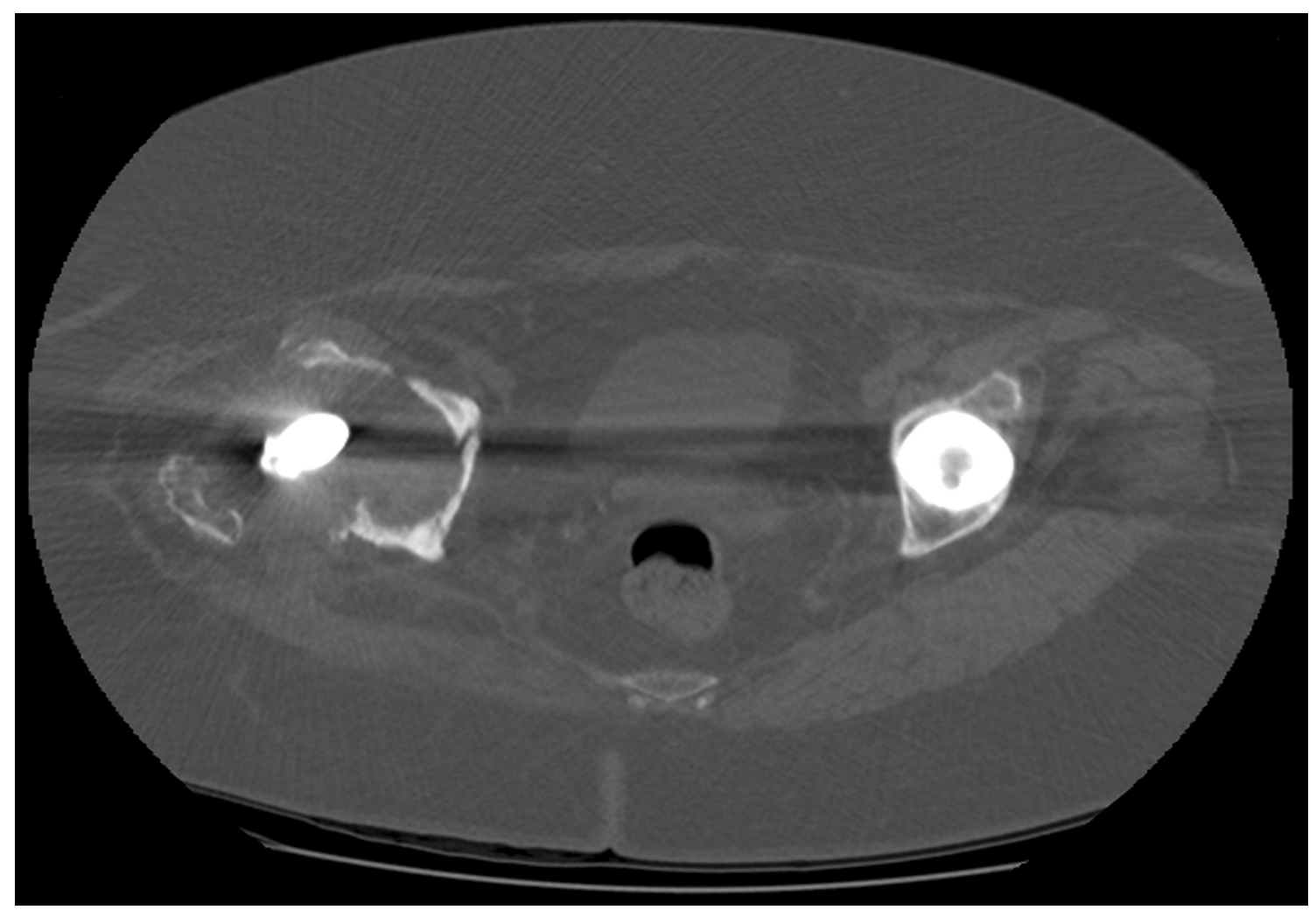

Figure 2: Preoperative axial view of CT scan showing acetabular deficit.

\section{Case 1}

A 67-year-old woman was referred to our Unit due to increasing right hip pain and decreased hip motion. She was treated with total hip arthroplasty 12 years previously and with revision surgery 2 years previously. 
The patient described pain in the right buttock and in the right inguinal area increasing with active and passive movements. At physical examination an antalgic gait and a flexion contracture of the right hip of 15 degrees were observed. Maximun flexion of the hip was 60 degrees with minimal internal and external rotation. Blood inflammatory markers were all within normal limits.

Radiographs and CT scans showed loosening of the acetabular component with partial intrapelvic migration and revealed a type IIIB acetabular defect according to the Paprosky classification [1] with a break in Kohler line. Total bone scintigraphy showed increased uptake in the right acetabulum.

The direct lateral approach was used. The femoral component was found to be stable. The acetabular component was removed without difficulty. Several samples for bacterial culture were performed during surgical procedure (they resulted negative for infection).

A large superolateral acetabular defect was present. After lavage of the acetabulum, two fresh-frozen femoral head allografts were impacted with use of impactors and hammer. Morselized allograft ( $40 \mathrm{gr}$.) was used to fill the smaller defects. A 60-mm uncemented porous-coated anatomic cup (Trilogy ${ }^{\oplus}$ Acetabular Hip System, Zimmer, Warsaw, Indiana USA) was placed with a press-fit with two supplementary acetabular screws. A 32-mm diameter ceramic femoral head was implanted on to stable stem. Full weightbearing ambulation was avoided for six-months after surgical procedure. At 1 year, postoperative radiographs showed bone grafts integration. At 1 year follow-up the patient was pain-free and ambulating without using supporting devices. (Figure 3 a,b,c)
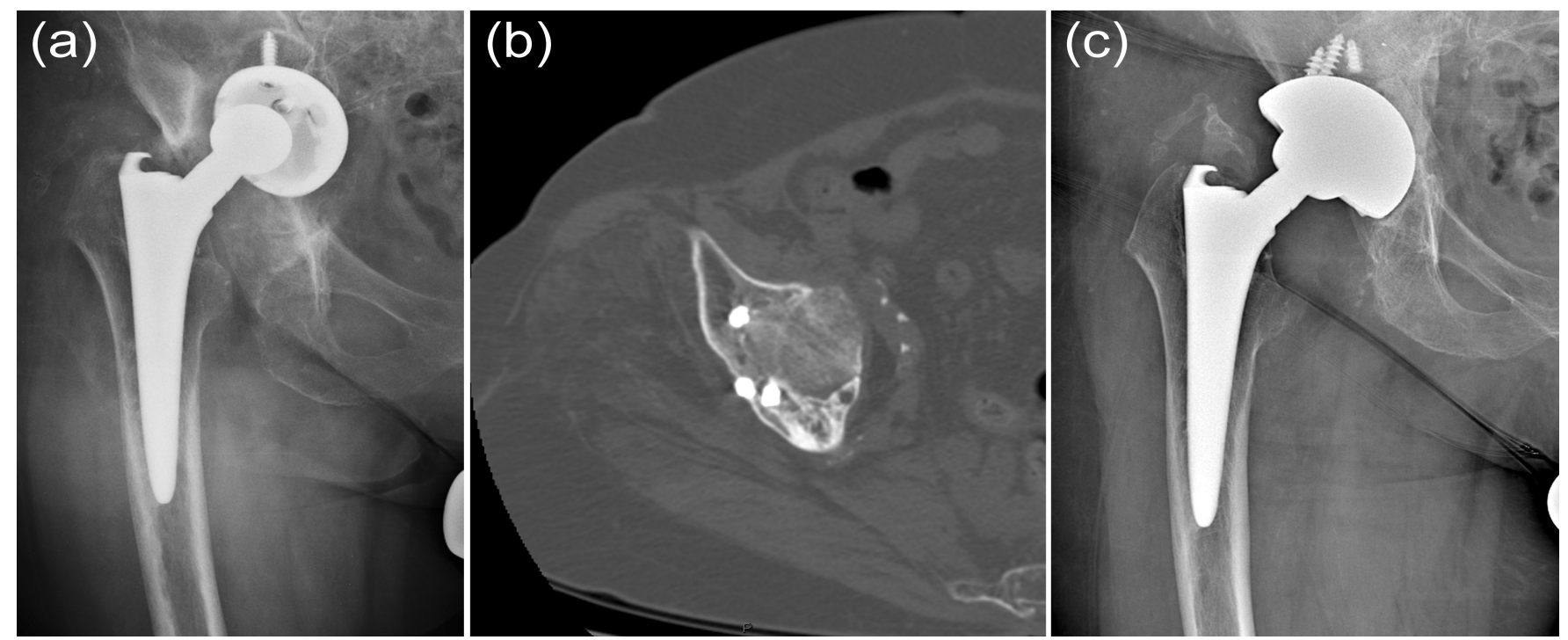

Figure 3: a. Preoperative radiograph showing right failed socket, with a superomedial migration of the acetabulum and a break in the Kohler line. b. Preoperative axial view of CT scan showing acetabular deficiency. c. Postoperative anterior-posterior radiographs showing initial remodelling of the femoral heads and well-fixed acetabular socket at 1 year follow-up.

\section{Discussion}

In acetabular reconstruction it is mandatory to evaluate the presence or absence of supporting structures such as medial wall, anterior and posterior columns and acetabular rim. Several techniques to manage pelvic bone defects have been described in the literature[2-6]. The main objectives in acetabular reconstruction are: stable fixation and anatomic restoration.

In our opinion the use of bone allograft is more physiologic and able to provide stability and mechanical support to the acetabular implant with appropriate use. We recommend cementless biologic fixation; in this way the bone stock can be restored and the patient may face, if necessary, future revision surgery. In the literature many authors do not share the use of cementless impaction grafting techniques due to a high risk of complications [7-9].

Van Haaren et al.[7] reported an overall survivorship of $72 \%$ at 7.2 years in their series of 71 revisions with impaction grafting technique. Hooten et al. [9] showed radiographic evidence of instability in $44 \%$ of 27 cementless revisions with bulk acetabular allograft at an average of 46 months follow-up.

We believe the use of impacted allograft may provide significant benefits in the medium and long term. The impaction procedure increases the medium-term stability of the construct while the biological behavior of the graft (remodeling with new bone formation and host-graft union) provides long-term stability. The bulk allografts are fresh-frozen femoral heads stored at minus $80{ }^{\circ} \mathrm{C}$. Cortical bone and cartilage are removed. The grafts are shaped in order to achieve larger diameter wider than the largest dimension of the bone defect. Then the grafts are placed in the acetabular defect and impacted. Subsequently the grafts are reamed to hold a hemispherical press-fit cup. Minor defects are filled with morselized bone graft. The hemispherical cup is impacted trying to achieve a partial direct contact with host bone in the acetabular weight-bearing area (De Lee and Charnley Zone I). The use of reinforcement devices requires a larger surgical approach and a more difficult surgical procedure. 
Structural allograft and primary press-fit cup techniques in our opinion increases long-term survival. Moreover structural allograft allows the placement of the cup at the correct anatomical level and the restoration of the lenght of the lower limb.

Traina et al. [10] reported a survival rate of $84 \%$ at 11 years in 23 patients with pelvic bone stock deficiency involving major columns underwent revision surgery with a cementless press-fit cup and a structural bone graft.

Comba et al. [11] reviewed the clinical and radiological results of 131 patients treated of acetabular revision with impacted bone allograft and cemented acetabular component. The survival rate was $95.8 \%$ at a minimum follow-up of two years and maximum of 13.1 years.

Both reported cases in our study showed good results: grafts integration and stable fixation of the socket were obtained at 1 year follow-up. At radiographs bridging trabeculae were present at the host bone-graft interface. In both cases the grafts were remodeled because resorption was limited to the area of the graft not contiguos to the host bone and the anatomy was restored.

Many studies described the use of reinforcement devices in association with bone grafts. The authors reported good results in severe acetabular bone deficiencies $[3,12,13]$.

Anyway in our opinion the use of reinforcement devices had some disadvantages. As mentioned above a larger surgical approach and a more demanding surgical procedure are necessary; furthermore the use of screws to fix the device to the pelvis can be dangerous.

Some authors used trabecular metal augments and shell in acetabular defect reconstruction [14]. The augment allows to fill the defect obviating the need for a structural allograft. Moreover the hemispheric cup combined with the augment increases the surface area for host-bone contact. This technique is more expensive than alternative techniques and cages and furthermore the acetabular shells are not fixed to the augments with screws and cement.

A reduced mechanical stress is fundamental to avoid resorption of the structural grafts and subsequent implant failure. Rehabilitation exercises are immediately started on the first post-surgical day. Patients use two crutches during walking for at least 45 days. Therefore we try to postpone full weight-bearing stresses at least five-six months after surgical procedure in order to preserve bone graft integration. During physiotherapy patients are educated to avoid adduction and forced externally rotation of the hip. Radiologic evidence of graft incorporation must be evident before prescribing weight-bearing.

\section{Conclusion}

Acetabular reconstruction with structural allograft and primary press-fit cup is a safe procedure in the treatment of acetabular deficiency. We recommend the use of impaction grafting techniques for Type III defects according to the Paprosky classification system [1].

\section{References}

1. Paprosky WG, Perona PG, Lawrence JM (1994) Acetabular defect classification and surgical reconstruction in revision arthroplasty. A 6-year follow-up evaluation. J Arthroplasty 9: 33-44.

2. Gill TJ, Sledge JB, Muller ME (2000) The management of severe acetabular bone loss using structural allograft and acetabular reinforcement devices. J Arthroplasty 15: $1-7$.

3. Kerboull M, Hamadouche M, Kerboull L (2000) The Kerboull acetabular reinfoircement device in major acetabular reconstructions. Clin Orthop 378: 155-68.

4. Berry DJ, Sutherland CJ, Trousdale RT, Colwell CW Jr, Chandler HP, et al. (2000) Bilobed oblong porous coated acetabular components in revision total hip arthroplasty. Clin Orthop 371: 154-60.

5. Emerson RH, Head WC (1993) Dealing with the deficient acetabulum in revision hip arthroplasty: the importance of implant migration and use of the jumbo cup. Semin Arthroplasty 1: 2-8.

6. Sporer SM, Paprosky WG (2006) The use of a trabecular metal acetabular component and trabecular metal augment for severe acetabular defects. J Arthroplasty 6: 83-6.

7. Van Haaren EH, Heyligers IC, Alexander FG, Wuisman PI (2007) High rate of failure of impaction grafting in large acetabular defects. J Bone Joint Surg Br 89: 296-300.

8. Kwong LM, Jasty M, Harris WH (1993) High failure rate of bulk femoral head allografts in total hip acetabular reconstructions at 10 years. J Arthroplasty 8: $341-6$. 9. Hooten JP Jr, Engh CA Jr, Engh CA (1994) Failure of structural acetabular allografts in cementless revision hip arthroplasty. J Bone Joint Surg Br 76: 419-22. 10. Traina F, Giardina F, De Clerico M, Toni A (2005) Structural allograft and primary press-fit cup for severe acetabular deficiency. A minimun 6-year follow-up study. Int Orthop 29:135-9.

11. Comba F, Buttaro M, Pusso R, Piccaluga F (2006) Acetabular reconstruction with impacted bone allografts and cemented acetabular components. a 2- to 13-year follow-up study of 142 aseptic revisions. J Bone Joint Surg Br 88: 865-9.

12. Bohm P, Banzhaf S (1999) Acetabular revision with allograft bone: 103 revisions with 3 reconstruction alternatives, followed for 0.3-13 years. Acta Orthop Scand 70: 240-9.

13. Saleh KJ, Jaroszynski G, Woodgate I, Saleh L, Gross AE (2000) Revision total hip arthroplasty with the use of structural acetabular allograft and reconstruction ring: a case series with a 10-year average follow-up. J Arthroplast 15: 951-8.

14. Nehme A, Lewallen DG, Hanssen AD (2004) Modular porous metal augments for treatment of severe acetabular bone loss during revision hip arthroplasty Clin Orthop Relat Res 429: 201-8. 